\title{
Implications of the blood-brain barrier in primary central nervous system lymphoma
}

\author{
Kristoph Jahnke, M.D., Nancy D. Doolittle, Ph.D., Leslie L. Muldoon, Ph.D., \\ AND EdWard A. NeUWelt, M.D. \\ Departments of Neurology, Medicine, Cell and Developmental Biology, and Neurosurgery, Oregon \\ Health \& Science University; and Veterans Administration Medical Center, Portland, Oregon
}

\begin{abstract}
$\checkmark$ The optimal treatment of primary central nervous system lymphoma (PCNSL), a rare form of extranodal nonHodgkin lymphoma, has yet to be defined. Whole-brain radiation therapy (WBRT) has limited efficacy as a single therapeutic modality and is associated with a high risk of delayed neurotoxicity. Methotrexate-based chemotherapy regimens yield poor drug penetration across the blood-brain barrier (BBB), thus necessitating administration of high doses with the concomitant risk of increased systemic and neurological toxicity. Combined-modality therapy (WBRT plus chemotherapy) can improve response and survival rates, yet it is associated with a high risk of neurotoxicity. The aim of chemotherapy in conjunction with BBB disruption is to maximize drug delivery to the brain and improve the agent's efficacy, while preserving neurocognitive function and minimizing systemic toxicity. Methotrexate-based chemotherapy regimens administered in conjunction with BBB disruption have shown promising results in PCNSL. Animal models of central nervous system lymphoma and drug neurotoxicity offer new possibilities to study the effects of various treatments on PCNSL and normal brain and can also help understand biological and pathophysiological aspects of this disease. Because the intact BBB is even less permeable to antibodies than it is to drugs, preclinical and clinical studies of monoclonal antibody delivery (for example, rituximab and ${ }^{90} \mathrm{Y}$ ibritumomab tiuxetan) to the brain in conjunction with BBB disruption offer a new possibility to make these novel treatments more efficient against PCNSL. Regarding the evaluation of more sensitive and specific diagnostic imaging tools, iron oxide-based contrast agents for magnetic resonance imaging have shown promise for better differentiation of PCNSL from other white matter diseases.
\end{abstract}

\section{KEY WORDS • primary central nervous system lymphoma • blood-brain barrier • methotrexate $\bullet$ rituximab $\bullet$ radiation therapy}

$\mathrm{T}$ HE incidence of PCNSL, an extranodal non-Hodgkin lymphoma confined to the CNS at the time of diagnosis, increased almost threefold from 1973 to 1984, ${ }^{23}$ but has stabilized recently. ${ }^{32}$ The optimal treatment for PCNSL has yet to be defined. Postoperative WBRT improves the median survival duration by up to 18 months, but responses are short-lived..$^{43}$ For methotrexate-based intravenous chemotherapy alone, a 5-year survival rate of up to $43 \%$ has been reported. ${ }^{66}$ Combined-modality therapy with high-dose methotrexate-based chemotherapy and WBRT improves response and survival rates compared with WBRT alone, but is associated with delayed neurotoxicity in 8 to $50 \%$ of patients, $1,16,29,61,79$ and the 5-year survival rates are 9 to $32 \% .^{1,7,16,64}$ Long-term survivors older than 60 years of age are at particular risk for experiencing late neurotoxicity; $1,7,67$ however, detailed neurocognitive outcomes and quality of life evaluations are rarely reported in published clinical trials.

Abbreviations used in this paper: $\mathrm{BBB}=$ blood-brain barrier; $\mathrm{CNS}=$ central nervous system; $\mathrm{CSF}=$ cerebrospinal fluid; CT = computed tomography; ICA = internal carotid artery; KPS = Karnofsky Performance Scale; $\mathrm{MR}=$ magnetic resonance; $\mathrm{MS}=$ multiple sclerosis; OHSU = Oregon Health \& Science University; $\mathrm{PCNSL}=$ primary $\mathrm{CNS}$ lymphoma; VA = vertebral artery; $\mathrm{WBRT}=$ whole-brain radiation therapy.
Osmotic BBB disruption-enhanced chemotherapy delivery for PCNSL has been ongoing at the OHSU since 1982, and is currently being performed at eight institutions worldwide that are participating in the International Blood-Brain Barrier Disruption Consortium. ${ }^{19}$ The BBB Program at OHSU is a translational clinical and research model with preclinical and clinical research teams reciprocally informing each other of new developments. The key paradigm for the BBB Program is that the efficacy of chemotherapy in brain tumors is disappointing overall, due in part to the fact that the BBB limits the passage of therapeutic agents to the tumor and surrounding brain. Exclusion of molecules from the brain by the BBB is based on molecular weight, charge, and lipid solubility. ${ }^{44}$ The goals of BBB disruption are as follows: 1) enhanced delivery of therapeutic agents to the brain, resulting in improved treatment outcomes; 2) preservation of neurocognitive function and quality of life; and 3) minimizing systemic toxicity. The BBB disruption technique is especially important in the delivery of high-molecular-weight agents, like monoclonal antibodies and radioimmunoconjugates ${ }^{44,48}$ that already are established treatment options in systemic lymphoma. ${ }^{24,60} \mathrm{We}$ present an overview and outlook regarding preclinical and clinical results in the treatment of PCNSL reported by investigators in the BBB 
Program, covering methotrexate-and carboplatin-based treatment regimens, the addition of monoclonal antibodies and radioimmunoconjugates to treatment regimens, neurotoxicity issues, and novel imaging techniques.

\section{Preclinical Studies}

\section{Delivery of Methotrexate-Based Chemotherapy to the Brain With and Without BBB Disruption}

Several observations regarding BBB disruption that have clinical implications have been made in animal studies. ${ }^{37,44,45}$ It has been shown that disruption of the BBB results in delivery of the respective agent throughout either the VA or ICA distribution. Variables that influence delivery are type, size, and location of the tumor. The concentration of methotrexate in brain and CSF can be substantially increased using BBB disruption. ${ }^{47,51,54,55,62,68-70}$ In normal dogs (Fig. 1), $4 \mathrm{mg} / \mathrm{m}^{2}$ methotrexate injected into the carotid artery without BBB disruption resulted in concentrations in the ipsilateral brain that were lower by a $\log$ factor than in the serum. However, 6 to 12 hours after disruption, cerebral concentrations exceeded those in serum by a $\log$ factor. The slower clearance of drug from the brain implies that therapeutic concentrations can be maintained for a longer period of time. With disruption of the VA, delivery to the brain areas it supplies increased 12- to 18 -fold. ${ }^{53}$

Similarly, in a rat glioma model there was a significant increase in delivery of methotrexate to the tumor, brain around the tumor, and brain distant to the tumor in the lesion-bearing hemisphere compared with uptake for tumor-bearing control animals in which the BBB was not disrupted. In the same study, administration of dexamethasone prior to BBB disruption resulted in a 40 to $60 \%$ decrease of methotrexate uptake in the tumor and, to a lesser extent, in the brain around and distant to the tumor. ${ }^{46}$ This study provided evidence that the BBB is partially intact in tumors, and that steroid drugs can alter the permeability of the disrupted BBB. Similar results were found in a small cell lung cancer xenograft model of CNS metastasis. ${ }^{49}$ These findings are important because a clear doseresponse relationship has been demonstrated in several studies of animal and human tumors (including in PCNSL), and even a $20 \%$ reduction in drug dose can have a dramatic impact on outcomes. ${ }^{18,25,27,35,36}$ A relationship between the degree of BBB opening and drug delivery in animals and the association of increasing dose intensity with increasing survival were demonstrated by our group in a nude rat model and in a human brain tumor xenograft model. ${ }^{37,72}$ Furthermore, a relationship between enhanced delivery of borinated compounds after BBB disruption and the efficacy of boron/neutron capture therapy in an animal brain tumor model has been reported. ${ }^{4}$

The exact timing of BBB disruption and drug administration is important. After BBB disruption, vascular permeability reaches maximum levels at 15 minutes after osmotic shock and then rapidly decreases, returning to preinfusion levels within 2 hours after disruption. These observations apply to small molecules such as methotrexate as well as to large molecules such as monoclonal antibodies. $37,44,45$

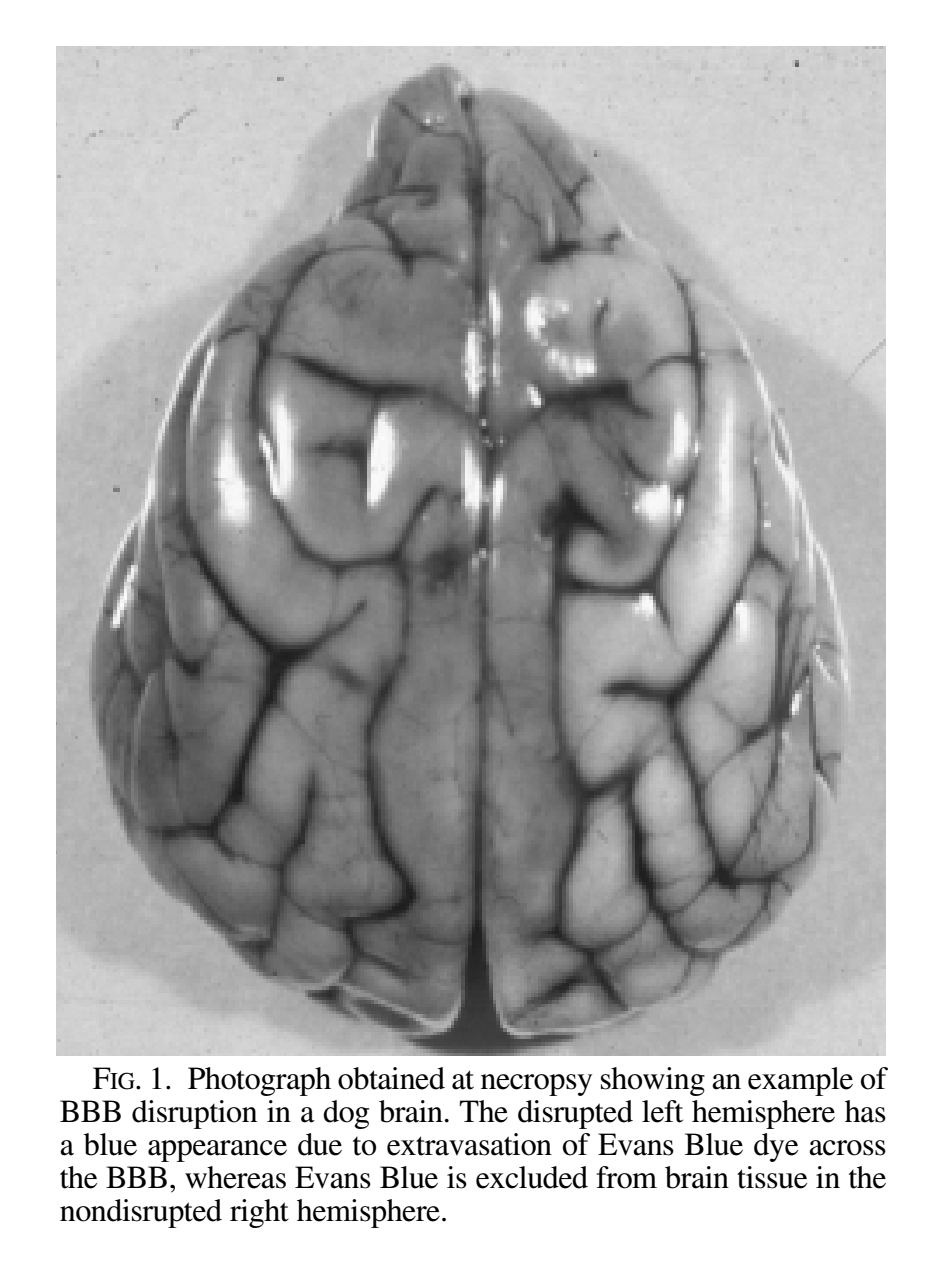

Impact of Radiation Therapy and Sequence of Treatment Modalities on Drug Delivery, Efficacy, and Neurotoxicity

A PCNSL is both radio- and chemosensitive. If radiotherapy is used, the sequence of radio- and chemotherapy influences the delivery of therapeutic agents to the brain and the degree of acute and delayed neurotoxicity. In a rodent model, two clinically relevant chemotherapy regimens (intraarterial methotrexate $\left[1 \mathrm{~g} / \mathrm{m}^{2}\right]$ and a combination of intravenous etoposide and intraarterial carboplatin) were given after BBB disruption. A single fraction of external-beam radiation ( $20 \mathrm{~Gy}$ ) was administered 30 days before (Group 1), concurrent with (24 hours prior; Group 2), or 30 days after (Group 3) chemotherapy. Radiation administered either before or concurrent with chemotherapy resulted in a significant $(\mathrm{p}<0.01)$ decrease in drug delivery to the brain compared with the levels found in animals that did not undergo brain irradiation. Furthermore, in Groups 1 and 2, seizures were observed in 26\% of the animals, and the mortality rate in animals receiving radiotherapy 30 days before chemotherapy was significantly $(\mathrm{p}=0.03)$ higher than in the control animals that did not receive radiation. ${ }^{73}$ Similarly, the long-term toxicity evaluation in the same rodent study confirmed that radiation before chemotherapy is the most toxic sequence, and additionally, that there were significant increases $(\mathrm{p}=$ $0.001,0.006$, and 0.013 , respectively, for the aforementioned treatment groups) in long-term toxic effects when 
comparing the treatment groups that received radiation plus chemotherapy with the control groups that received only radiation or chemotherapy. ${ }^{72}$

There is evidence that the sequence of therapy modalities also influences treatment outcomes. In nude rats bearing the human lung cancer cell line LX-1, cranial radiotherapy (20 Gy, single fraction) was administered either before, concurrent with, or after BBB disruption-enhanced treatment with the antibody-targeted chemotherapy agent BR96-doxorubicin. Results indicated that disruption-enhanced delivery of immunoconjugates is more effective in combination with WBRT. Moreover, administration of BR96-doxorubicin before WBRT significantly increased survival compared with the treatment group that received WBRT and immunochemotherapy concurrently $(p<0.05) .{ }^{71}$ Although these findings were observed in a lung cancer and not in a PCNSL model, the results were later confirmed in the clinical setting in patients with PCNSL. Participants who underwent WBRT before BBB disruption-enhanced chemotherapy had a significantly decreased median survival duration compared with patients who received BBB disruption chemotherapy initially (see Clinical Studies for details). ${ }^{14,58}$

\section{New Animal Models for PCNSL and Neurotoxicity Studies}

Because the optimal clinical treatment for PCNSL remains controversial, animal models that closely mimic the clinical situation could prove useful for evaluating biological, pathophysiological, and therapeutic aspects of PCNSL. In the past, animal models of PCNSL have mainly been focused on T-cell models; ${ }^{3,81,90}$ however, T-cell PCNSL is rare and accounts for less than 5\% of all tumors of this type. ${ }^{83}$ The only established human xenograft Bcell model of PCNSL in which human B lymphoma cells have been used focused on pathological aspects of the disease, and interventional studies were not performed. ${ }^{77}$ Thus, there is a need for a new B-cell lymphoma model to generate preclinical data for possible translation to clinical practice.

The investigators in the BBB Program at OHSU recently released preliminary data on a new B-cell PCNSL model in which the human MC116 lymphoma cell line was used. ${ }^{84}$ In this model, female nude rats were pretreated with WBRT, compared with a group that received no pretreatment, 24 hours before intracerebral (right caudate nucleus) inoculation of MC116 human B-cell lymphoma cells. Tumor growth and permeability were evaluated using MR imaging at various time points ranging from 1 to 4 weeks after tumor inoculation. To evaluate tumor response, selected rats were treated with WBRT $(20 \mathrm{~Gy})$ or high-dose intravenous methotrexate $\left(3 \mathrm{~g} / \mathrm{m}^{2}\right)$. The rat brains were assessed 3 to 5 weeks after tumor implantation for lesion volumes and immunoreactivity. The MC116 cells formed infiltrative CD20- and CD45-positive brain tumors that spread through the cortex in the inoculated hemisphere, along the meninges, and into the ventricles. The tumor growth rate and pattern of infiltration were independent of preinoculation irradiation status. The MR images demonstrated that tumor permeability was heterogeneous, but generally demonstrated gadolinium enhancement at the inoculation site in the cortex and in the ventricles. A single treatment with WBRT markedly reduced
MR imaging enhancement and tumor volume, but intravenous methotrexate was not effective. In summary, this human B-cell PCNSL model in rodents displays growth, imaging, and immunological characteristics that closely resemble human PCNSL. This model will be useful for evaluating chemotherapy and immune-targeted therapies for PCNSL. A study testing the efficacy of methotrexateand rituximab-based immunochemotherapy with or without $\mathrm{BBB}$ disruption is currently in progress.

\section{Clinical Studies}

\section{The Clinical BBB Disruption Technique and Treatment Protocols for PCNSL}

The care of patients undergoing BBB disruptionenhanced chemotherapy has been described in detail elsewhere. ${ }^{21,58}$ Briefly, the BBB disruption treatment is done on 2 consecutive days every 4 weeks for up to 1 year. The care of patients treated with this technique requires a multidisciplinary team approach. The BBB disruption is performed after induction of general anesthesia to ensure patient comfort and safety. A femoral artery is accessed, and an intracranial artery (ICA or VA) is catheterized. On the 1 st day one artery is infused, followed by a different artery on the 2nd day. Hypertonic (25\%), warmed mannitol is delivered at a predetermined flow rate of 3 to $12 \mathrm{ml} / \mathrm{sec}$ ond into the chosen artery for 30 seconds. After administration of mannitol, the intraarterial chemotherapy agent(s) are infused, each for 10 minutes. Intravenous chemotherapy is begun directly after induction of general anesthesia to allow time for the drug to be delivered to the tumor while the BBB is open. Immediately after the mannitol infusion, nonionic contrast dye is administered intravenously. Following completion of chemotherapy, the patient undergoes a CT brain scan. ${ }^{22}$ Contrast enhancement in the disrupted territory of the brain is compared to that seen in the nondisrupted territory. The degree of disruption is graded as nil, moderate, good, and excellent, according to the scale published by Roman-Goldstein, et al. ${ }^{75}$ (Fig. 2 shows examples of a good disruption).

At OHSU, two different chemotherapy regimens for PCNSL have been used in the past. Protocol I, which was used from 1982 until 1993,,$^{58}$ consisted of intraarterial administration of $2.5 \mathrm{~g} /$ day methotrexate and intravenous administration of $15 \mathrm{mg} / \mathrm{kg}$ cyclophosphamide on each of

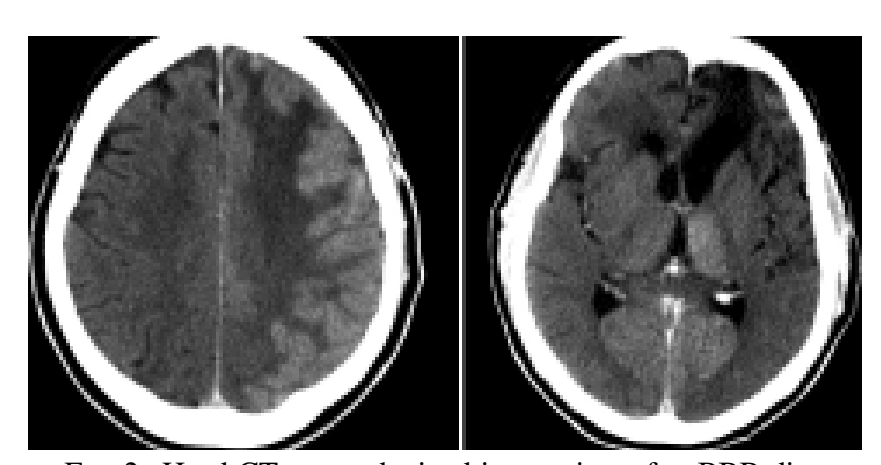

FIG. 2. Head CT scans obtained in a patient after BBB disruption of the left ICA (left) and VA (right) distributions. The degree of disruption was graded as good, as evidenced by bright contrast enhancement in each arterial distribution. 
K. Jahnke, et al.

TABLE 1

Literature review of patient characteristics and treatment outcomes in clinical studies

for PCNSL in which chemotherapy was used alone or in conjunction with WBRT*

\begin{tabular}{|c|c|c|c|c|c|c|c|}
\hline Authors \& Year & Tx Modality & $\begin{array}{c}\text { No. of } \\
\text { Patients }\end{array}$ & $\begin{array}{c}\text { Median } \\
\text { Age (yrs) }\end{array}$ & $\begin{array}{c}\text { Median } \\
\text { KPS }\end{array}$ & $\begin{array}{l}\text { Overall } \\
\text { RR }(\%)\end{array}$ & $\begin{array}{c}\text { Median } \\
\text { OS (mos) }\end{array}$ & $\begin{array}{c}5-\mathrm{Yr} \\
\mathrm{OS}(\%)\end{array}$ \\
\hline Abrey, et al., 1998 & chemo + WBRT & 31 & 59 & NR & $87.1 \dagger$ & $42 \div$ & 22.3 \\
\hline Blay, et al., 1998 & $\begin{array}{l}\text { chemo, WBRT, } \\
\text { combined chemo } \\
\text { + WBRT }\end{array}$ & 226 & 61 & NR & NR & 16 & 19 \\
\hline Brada, et al., 1998 & chemo + WBRT & 31 & 51 & 60 & $67 \dagger$ & 23 & 34 \\
\hline Sandor, et al., 1998 & chemo & 14 & 57 & NR & 100 & $\mathrm{nr}$ & $68.8 \S$ \\
\hline McAllister, et al., 2000 & chemo + BBBD & 74 & NR\| & NR\| & $65 \dagger$ & 40.7 & 42 \\
\hline DeAngelis, et al., 2002 & chemo + WBRT & 102 & 56.5 & 80 & 94 & 36.9 & 32 \\
\hline Batchelor, et al., 2003 & chemo & 25 & 60 & 80 & 74 & $\geq 22.8$ & NR \\
\hline Pels, et al., 2003 & chemo & 65 & 62 & 70 & 71 & 50 & 43 \\
\hline Poortmans, et al., 2003 & chemo + WBRT & 52 & 51 & 70 & 81 & 46 & $58 * *$ \\
\hline
\end{tabular}

$* \mathrm{BBBD}=\mathrm{BBB}$ disruption; chemo = chemotherapy; $\mathrm{nr}=$ not reached; $\mathrm{NR}=$ not reported; OS = overall survival; $\mathrm{RR}=$ response rate; $\mathrm{Tx}=$ treatment.

$\dagger$ Complete remissions only.

\$ Median cause-specific survival.

$\S$ At 57 months.

II In this cohort, 36 patients (49\%) were younger than 60 , and $38(51 \%)$ were 60 years of age or older. The KPS score was greater than 70 in 46 patients $(62 \%)$, and 70 or less in 26 patients $(35 \%)$

** This value represents the 3 -year survival.

2 consecutive days, followed by 14 days of $100 \mathrm{mg} /$ day orally administered procarbazine given monthly for 1 year. In 1993, Protocol II eliminated procarbazine to permit the use of granulocyte colony-stimulating factor. Protocol II consisted of intraarterial administration of $2.5 \mathrm{~g} /$ day methotrexate, $150 \mathrm{mg} / \mathrm{m}^{2} /$ day intravenous or intraarterial etoposide phosphate, and $500 \mathrm{mg} / \mathrm{m}^{2} /$ day intravenous cyclophosphamide on each of 2 consecutive days, repeated monthly for 1 year. Protocol II used $5 \mathrm{mg} / \mathrm{kg}$ granulocyte colony-stimulating factor for 7 to 10 days, starting on Day 3. Protocol II was used until 2005, when two new PCNSL treatment protocols, one for first-line treatment and one for relapsing disease, opened for enrollment. Both protocols use rituximab and carboplatin, two drugs that have shown promising results in the treatment of extracerebral lymphoma. For newly diagnosed PCNSL, 2.5 g/day methotrexate and $200 \mathrm{mg} / \mathrm{m}^{2} /$ day carboplatin (total dose 400 $\mathrm{mg} / \mathrm{m}^{2}$ ) are given with BBB disruption on each of 2 consecutive days. Additionally, $375 \mathrm{mg} / \mathrm{m}^{2}$ rituximab is administered intravenously within 24 hours prior to the first BBB disruption of each monthly course. In the protocol for relapsed disease, rituximab and carboplatin are administered as in the first-line protocol. In addition, $330 \mathrm{mg} / \mathrm{m}^{2} /$ day cyclophosphamide (total dose $660 \mathrm{mg} / \mathrm{m}^{2}$ ) and 200 $\mathrm{mg} / \mathrm{m}^{2} /$ day etoposide/etoposide phosphate (total dose 400 $\mathrm{mg} / \mathrm{m}^{2}$ ) are given intravenously. In all regimens in which carboplatin is used, patients receive high-dose sodium thiosulfate to protect against carboplatin-induced ototoxicity. ${ }^{20}$

\section{The Importance of Drug Delivery in the Treatment of PCNSL}

Combination chemotherapy for systemic aggressive non-Hodgkin lymphoma results in probable cure for a considerable number of patients; however, it has only very modest efficacy in treating PCNSL when administered intravenously ${ }^{86}$ It was already suggested in the early $1980 \mathrm{~s}$ that the BBB interferes with drug delivery to the CNS. This was hypothesized when two patients with systemic lymphoma in remission experienced CNS involvement while undergoing treatment with multidrug chemotherapy. ${ }^{30}$ The BBB normally excludes ionized, water-soluble drugs with molecular weights greater than $180 \mathrm{D}$ from entering the brain, and most chemotherapeutic agents weigh between 200 and $1200 \mathrm{D} .{ }^{45}$ Furthermore, the intact BBB is even less permeable to antibodies than to drugs. In a study in which the CSF penetration of the monoclonal antibody trastuzumab was investigated in six patients with breast cancer and brain metastases, the serum/CSF ratio of trastuzumab was $420: 1$, indicating poor penetration of the agent. However, with impaired integrity of the BBB and blood-CSF barrier, smaller ratios (76:1 in two patients after WBRT and 49:1 in two patients with meningeal carcinomatosis) were observed. ${ }^{85}$

In PCNSL, administration of high-dose intravenous methotrexate has been performed in an attempt to improve delivery across the BBB and blood-CSF barrier. The CSF penetration of intravenously administered methotrexate in humans is dose dependent, as was demonstrated in several studies. ${ }^{10,12,28,41,42,87}$ Nevertheless, cytotoxic CSF levels $\left(>10^{-6} \mathrm{M}\right)$ were not achieved with a dose of $0.5 \mathrm{~g} / \mathrm{m}^{2}$, and only $44 \%$ of patients treated with $2.5 \mathrm{~g} / \mathrm{m}^{2}$ showed evidence of cytotoxic CSF concentrations ${ }^{87}$ A dose of $5 \mathrm{~g} / \mathrm{m}^{2}$ in children resulted in cytotoxic levels in 66 and $81 \%$ of patients in two separate studies ${ }^{41,42}$ However, it is unclear whether CSF levels after intravenous administration of methotrexate correspond to intratumoral concentrations and levels in brain, brain around tumor, and normal brain. Scarce clinical pharmacokinetic data are available regarding these compartments, which are important in highly infiltrative brain tumors such as PCNSL. Researchers in a recent study used microdialysis to evaluate drug levels in extracellular fluid in four patients with high-grade glioma after intravenous administration of methotrexate. ${ }^{63}$ Patients in whom the probe was located in contrast-enhancing tumor had higher methotrexate peak concentrations in extracellular fluid compared with patients in whom the probe was placed in nonenhancing tissue next to the enhancing 


\section{Implications of the blood-brain barrier in primary}

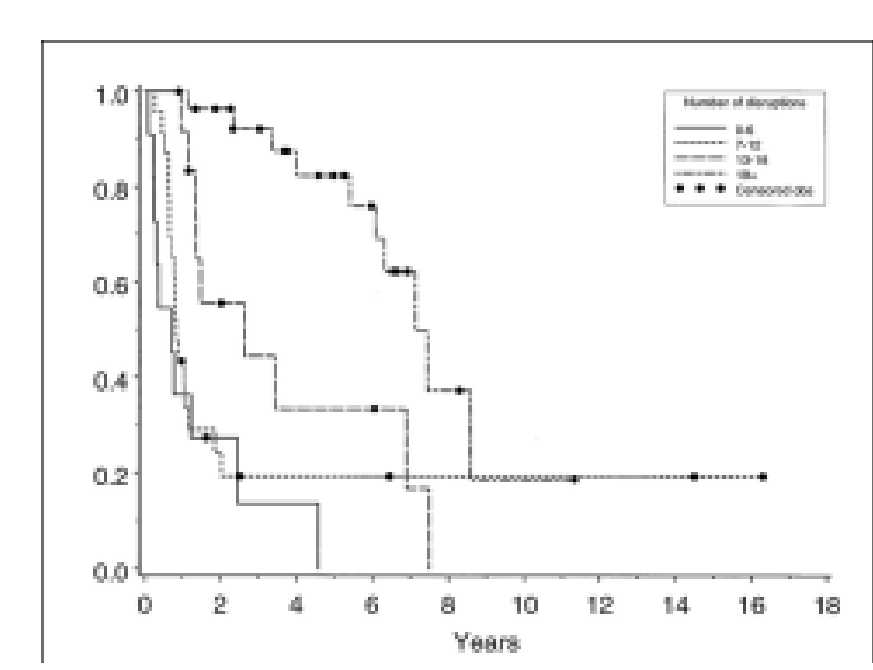

FIG. 3. Kaplan- Meier plot showing overall survival rates in 74 patients with PCNSL treated with BBB disruption-enhanced delivery of methotrexate. Results confirm the importance of dose intensity in the treatment of CNS neoplasms. In this study, the number of disruptions was used as a surrogate marker for dose intensity. The values on the $\mathrm{y}$ axis represent the relative survival. Obs = observations. Reprinted with permission from Kraemer DF, Fortin D, Doolittle ND, Neuwelt EA: Association of total dose intensity of chemotherapy in primary central nervous system lymphoma (human non-acquired immunodeficiency syndrome) and survival. Neurosurgery 48: 1033-1040, 2001.

tumor $(189 \pm 6 \mu \mathrm{M}$ compared with $10.4 \pm 0.4 \mu \mathrm{M})$. These observations indicate that intracerebral methotrexate distribution is associated with BBB integrity as assessed by measuring contrast enhancement on MR images and CT scans. As the tumor shrinks in response to chemotherapy, the BBB integrity is restored. This often results in only a transient tumor response, due to remaining viable tumor cells. In two patients with PCNSL who were initially treated with chemotherapy, significant changes in permeability in the region of the tumor were observed. Within 5 weeks of the start of treatment, permeability values as determined by the washout rate of the radioactive tracer ${ }^{68} \mathrm{Ga}$-ethylenediaminetetraacetic acid reached the levels found in normal brain. ${ }^{65}$

Intraventricular and intrathecal methotrexate administration lead to therapeutic CSF levels, ${ }^{31}$ but concentrations are inhomogeneous..$^{82}$ Furthermore, intrathecal administration can only achieve therapeutic levels in the superficial 2 to 3 $\mathrm{mm}$ of CNS parenchyma located beyond the subarachnoid space. ${ }^{6,34}$ Increased rates of neurotoxicity in patients treated with intravenous methotrexate or WBRT were reported when additional intrathecal methotrexate was given. ${ }^{9}$

Clinical studies in which researchers have documented the delivery of methotrexate and its efficacy when used in conjunction with osmotic BBB modification date back to the late 1970 s and early 1980 s..$^{50,52}$ Thirty consecutive patients with PCNSL who received the same BBB disruption chemotherapy regimen (Protocol I, using intraarterial methotrexate plus BBB disruption; see earlier description) between 1982 and 1989 and were followed until 1990 were divided into two groups. The 13 patients in Group 1 had received first-line treatment with WBRT and were enrolled in Protocol I after tumor progression or recurrence. The 17 patients in Group 2 were enrolled in Protocol I immediately and received WBRT only in the case of an incomplete tumor response or disease progression. The difference in median survival duration (17.8 months for Group 1 and 44.5 months for Group 2) was statistically significant $(p=0.039)$. Furthermore, a plateau in survival curves and manageable toxicity in the patients in Group 2 suggested that BBB disruption plus chemotherapy can produce long-term remission with improved survival, acceptable rates of morbidity and mortality, and preservation of cognitive function by sparing these patients WBRT with its associated risk of neurocognitive decline. ${ }^{58}$

These favorable results were confirmed in studies with a larger number of patients and longer follow-up period. ${ }^{14,39}$ In the study by McAllister, et al.,${ }^{39}$ consecutive patients with PCNSL whose first-line treatments were Protocols I and II between 1982 and 1997 (see earlier descriptions) were evaluated for tumor response and neurocognitive sequelae. The median follow-up duration after the first BBB disruption treatment was 6.2 years (range 6 months-16.3 years). The complete response rate was $65 \%$ (48 patients), and after 1 year the disease in $36(75 \%)$ of these patients continued to be in complete remission. An estimated 5-year survival rate of $42 \%$ and a median survival duration of 40.7 months were reported. These outcomes compare well with those reported by other investigators (Table 1) and substantiate the claim of a favorable outcome in patients with PCNSL treated with BBB disruption-enhanced chemotherapy.

\section{The Importance of Dose Intensity in the Treatment of PCNSL}

A relationship between the degree of $\mathrm{BBB}$ opening and drug delivery and the association of increased dose intensity with improved survival has been demonstrated in preclinical studies by our group and others., ${ }^{4,37,74}$ The importance of dose intensity in the clinical setting has remained an open question in the treatment of both systemic solid tumors and primary brain tumors. It has been hypothesized that dose and the use of combination chemotherapies, that is, the concept of "summation dose intensity," influences the outcomes of malignant diseases. ${ }^{26}$ Until recently, however, dose intensity (for example, the concept of high-dose chemotherapy with autologous stem cell support) had proven to be effective in several hematological malignancies but not in solid tumors, especially brain tumors. ${ }^{80}$ We believe that the key to successful treatment of brain tumors is drug delivery to the lesion-infiltrated brain around the tumor, with a relatively intact BBB. ${ }^{89}$ Therefore, the demonstration of a statistically significant connection between dose intensity and improved survival in an infiltrative and chemoresponsive primary brain tumor like PCNSL with variable integrity of the BBB, accomplished using BBB disruption-enhanced chemotherapy delivery, was important for establishing new paradigms in the management of infiltrative primary brain tumors (Fig. 3) ${ }^{35}$ In this study, in which 74 patients with PCNSL who underwent first-line treatment with BBB disruption-enhanced chemotherapy (the clinical characteristics of this cohort are described in McAllister, et al. ${ }^{39}$ ) were assessed, either the number of intraarterial infusions 
in conjunction with $\mathrm{BBB}$ disruption or a cumulative score derived from the quality of the BBB disruption visualized using cerebral CT scans was used as surrogate markers for dose intensity. In multivariate analysis, the number of disruptions was significantly associated with prolonged survival $(\mathrm{p}=0.04)$, and the cumulative disruption score approached statistical significance $(p=0.066)$. Survival bias does not fully explain these associations because increased dose intensity also resulted in improved survival in patients with complete tumor response.

\section{Preservation and Evaluation of Neurocognitive Function in Patients Treated With BBB Disruption-Enhanced Chemotherapy}

Treatments for PCNSL in which WBRT is used can prolong survival; however, there is extensive evidence that a substantial risk of neurocognitive decline is associated with WBRT, and it increases with advanced age and prolonged disease-free survival. ${ }^{2,33}$ In many clinical trials of PCNSL treatments, investigators have reported high rates of therapy-related neurotoxicity, ${ }^{1,67}$ which seems especially pronounced with combined-modality therapy (WBRT plus chemotherapy). In one study in which chemotherapy and WBRT were used in newly diagnosed PCNSL, 12 patients $(15 \%)$ experienced severe delayed neurotoxicity, and eight of them died. ${ }^{16}$ Regarding treatment-related neurotoxicity, neurological sequelae usually develop months or years after therapy. ${ }^{33}$ Cognitive function can only be accurately evaluated in the absence of tumor because it is impossible to differentiate treatmentrelated neurotoxicity from the tumor's effects on cognition. ${ }^{15}$ As a result, the incidence of neurocognitive decline is directly proportional to the percentage of patients with disease-free survival. ${ }^{17}$ With new treatment approaches, long-term disease-free survival in PCNSL has increased, and thus thorough assessment and preservation of neurocognitive function is a very important aspect in the care of patients with PCNSL.

In clinical trials published by investigators in the BBB Program so far, the results indicate preservation of neurocognitive function during and after PCNSL treatment. ${ }^{14,39,58}$ As part of clinical trials within the BBB Program, patients undergo extensive neuropsychological evaluation. The rationale for the choice of specific neuropsychological tests and their appropriate use in cognitive assessment of patients with brain tumors have been reported previously..$^{13}$ In the largest study in which longterm results have been evaluated in patients with PCNSL treated with BBB disruption, ${ }^{39}$ detailed neuropsychological data were available in $86 \%$ of the 36 patients who were in continued, complete remission 1 year posttreatment. None of the patients demonstrated cognitive loss on neuropsychological tests and clinical examinations. Patients either remained at their baseline cognitive level or showed improvement. This series includes eight patients older than 60 years of age in whom stability in neurocognitive function was documented after completion of treatment. These results are in contrast to those reported in elderly patients treated with combined-modality therapy. ${ }^{7}$ In one series, ${ }^{1}$ no patient older than 60 years of age at diagnosis remained free of dementia for more than 48 months. In summary, the results of the PCNSL studies published by investigators in the BBB Program demonstrate that the combination of favorable survival data and preservation of neurocognitive function is a key feature of BBB disruption-enhanced chemotherapy delivery.

Much controversy has surrounded the question whether changes on MR imaging correlate with neurocognitive function in patients with brain tumors. Unfortunately, studies comparing imaging changes and cognitive function over a long period of survival are rare. ${ }^{40}$ In one study, Roman-Goldstein, et al., ${ }^{76}$ evaluated 15 patients with brain tumors (among them nine with PCNSLs) who had a complete tumor response after undergoing $\mathrm{BBB}$ disruption with intraarterial chemotherapy. In some patients, new abnormalities were found to have developed on MR imaging. These patients, however, maintained the same level of cognitive and neurological functioning, and it was concluded that MR imaging findings do not correlate with the results of neurocognitive testing.

Similarly, 16 patients with PCNSL were followed after complete remission of disease was achieved using a methotrexate-based treatment regimen in conjunction with BBB disruption. ${ }^{56}$ Cognitive testing and MR images or CT scans were done for each patient before treatment and at its completion after 12 months. Thereafter, the same studies were done in nine of the 16 patients with complete remission, who were followed for a median of 55 months. Although a significant association $(p<0.028)$ was found between neurocognitive data and abnormalities on $\mathrm{T}_{2}-$ weighted MR images or low-attenuation areas on CT scans before chemotherapy, there was no correlation at completion of the treatment. In seven patients a new abnormality developed by the end of treatment, and was revealed on $\mathrm{T}_{2}$-weighted MR images or as a low-attenuation area on CT scans, whereas in 15 patients stable, decreased, or resolved baseline imaging abnormalities were demonstrated. Although cognitive impairment was common before they started therapy, all patients' cognitive function had improved significantly $(p<0.005)$ by the end of treatment. The data from this study confirm that neither BBB disruption before delivery of chemotherapy nor changes on imaging studies are associated with a decrease in cognitive function in patients with PCNSL in complete remission. This is in accordance with data in children that also support the suggestion that imaging changes are not associated with neurological dysfunction, and that these changes are frequently overinterpreted in relation to their clinical significance. ${ }^{8}$

\section{Toxicity of BBB Disruption-Enhanced Chemotherapy}

In Table 2 we report the toxicities leading to adverse events recorded for 74 patients with PCNSL who were treated with intraarterial chemotherapy in conjunction with $\mathrm{BBB}$ disruption. ${ }^{39}$ All four deaths within 30 days after the procedure were related to infections, and three of them occurred before the routine use of granulocyte colony-stimulating factor. Procedure-related toxicities include those specifically related to angiography (such as strokes and arterial injuries) and events related to BBB disruption procedures or the drugs used to induce general anesthesia. Seizures were the most common BBB disruption-related neurological adverse events and occurred during 6 to $8 \%$ of the procedures. They were not associat- 
TABLE 2

Toxicity of methotrexate-based intraarterially delivered chemotherapy in conjunction with $B B B$ disruption in 74 patients with PCNSL*

\begin{tabular}{|c|c|c|}
\hline Toxicity & $\begin{array}{l}\text { No. of Events } \\
\text { (no. of patients) }\end{array}$ & $\begin{array}{c}\text { Incidence } \\
\text { (\% of procedures) }\end{array}$ \\
\hline \multicolumn{3}{|l|}{ neurological } \\
\hline $\begin{array}{c}\text { reversible neurological } \\
\text { deficit }(>48 \mathrm{hrs})\end{array}$ & $11(7)$ & 1 \\
\hline $\begin{array}{l}\text { asymptomatic extracranial } \\
\text { arterial injury }\end{array}$ & $10(8)$ & 0.9 \\
\hline stroke & $5(5)$ & 0.5 \\
\hline status epilepticus & $1(1)$ & 0.1 \\
\hline cerebral herniation & $1(1)$ & 0.1 \\
\hline death w/in 48 hrs & 0 & 0 \\
\hline death w/in 30 days & 4 & 0.4 \\
\hline \multicolumn{3}{|l|}{ nonneurological } \\
\hline neutropenic fever & 21 & 3.6 \\
\hline deep venous thrombosis & 17 & 1.8 \\
\hline $\begin{array}{l}\text { thrombocytopenia or anemia } \\
\text { requiring transfusions of platelets } \\
\text { or packed redblood cells }\end{array}$ & 14 & 1.6 \\
\hline pneumonia & 3 & 1.3 \\
\hline septicemia (nonneutropenic) & $9(8)$ & 0.8 \\
\hline dysrhythmia & 3 & 0.3 \\
\hline pulmonary embolism & 2 & 0.3 \\
\hline femoral arterial thrombosis & 1 & 0.2 \\
\hline respiratory arrest & 1 & 0.1 \\
\hline pneumothorax & 1 & 0.1 \\
\hline urethral tear w/ bleeding & 1 & 0.1 \\
\hline femoral artery injury & 1 & 0.1 \\
\hline dose reductions & 7 & \\
\hline \multicolumn{3}{|l|}{ late complications } \\
\hline $\begin{array}{l}\text { orthopedic (long bone fractures, } \\
\text { avascular necrosis) }\end{array}$ & $8(7)$ & \\
\hline ophthalmological $\dagger$ & $12(12)$ & \\
\hline acute myelogenous leukemia & $1(1)$ & \\
\hline dementia & 0 & \\
\hline
\end{tabular}

* Adapted from McAllister, et al. (1074 procedures).

$\uparrow$ Includes more than $50 \%$ decrease in visual acuity (four cases), asymptomatic macular degeneration (two cases), and new cataracts (two cases).

ed with permanent neurological deficits. Three of five patients who suffered strokes after BBB disruption procedures showed no evidence of long-term cognitive deficits on neuropsychological tests. Transient neurological deficits were generally associated with good to excellent grades of BBB disruption, and the patients ultimately returned to baseline status. One patient experienced tonsillar herniation after a BBB disruption procedure, and that individual remains alive, with a KPS of $100 \%$. The nonneurological toxicities associated with this treatment were primarily related to administration of chemotherapy and corticosteroid drugs. These toxicities included neutropenic fever, anemia requiring transfusions, septicemia, and pneumonia.

\section{New MR Imaging Agents for the Differential Diagnosis of Brain Lesions}

The differential diagnosis of PCNSL can be difficult because this tumor can present with radiological, clinical, and laboratory similarities to other brain lesions, especially MS. The prognosis for both PCNSL and MS relies to a significant extent on the patient's neurological condition prior to the start of therapy; early diagnosis is the key for successful treatment. Thus, MR imaging agents that aid in the differential diagnosis of PCNSL can potentially reduce the interval from symptom presentation to diagnosis and may therefore improve clinical outcomes.

So-called ultrasmall superparamagnetic iron oxide nanoparticles have shown potential in the imaging of brain tumors. ${ }^{38,57,59,88}$ Gadolinium has a short plasma half-life of approximately 90 minutes, but ferumoxtran-10, one of the new investigational nanoparticles being assessed for use as MR imaging contrast agents, has a long half-life of 24 to 30 hours. In contrast to gadolinium, the ferumoxtran-10 molecule is much larger; it is the size of a small virus. Moreover, as opposed to gadolinium, ferumoxtran-10 is endocytosed by phagocytes like macrophages and by glial cells, ${ }^{57,88}$ whereas gadolinium does not enter cells. This cell-specific uptake difference may allow lesion enhancement even with a small area of BBB leakage if the BBB opening permits iron oxide particles to cross slowly, because of the long plasma half-life of ferumoxtran- 10 .

In a recently published study, ${ }^{38} 23$ patients with various CNS lesions (among them five patients with PCNSL and seven with MS) underwent brain MR imaging with and without gadolinium, which was followed at a mean interval of 10 days by a ferumoxtran-10 scan. The ferumoxtran-10 showed different enhancement patterns in a variety of CNS lesions. In PCNSL, ferumoxtran-10 may show a larger extent of the tumor or even additional lesions that are not shown with gadolinium (Fig. 4). Most important for the differential diagnosis of PCNSL, patients with MS demonstrated less or no enhancement with ferumoxtran10 compared to gadolinium. Thus, the differential diagnosis of PCNSL versus MS (and possibly other inflammatory CNS lesions) can be improved, thereby possibly shortening the interval from the onset of symptoms to the establishment of a diagnosis.

\section{Summary and Future Directions}

Tumor recurrence remains common after initial therapy for PCNSL, underscoring the need for novel treatment strategies to induce durable complete remissions. Over the past 25 years, it has been demonstrated in various studies that BBB disruption-enhanced methotrexate-based chemotherapy is an effective treatment for PCNSL that obviates or at least postpones the need for WBRT for a substantial number of patients. Moreover, the potential for long-term survival with preserved cognitive function and the importance of dose intensity has been demonstrated. Although comparisons of published case series are subject to potential biases, in most studies reported so far in which either chemotherapy alone or combined-modality therapy was used, researchers applied patient inclusion criteria similar to those used for the BBB group's studies (Table 1 ). The 5-year survival rate of $42 \%$ and the median survival duration of 40.7 months reported by McAllister, et al. ${ }^{39}$ compare well with other recent benchmark studies. In one study in which high-dose methotrexate-based chemotherapy followed by WBRT was used, a median survival duration of 36.9 months with a 5-year survival rate of $32 \%$ was reported; however, a high rate of neurotoxicity was observed (Table 1). ${ }^{16}$ In the second study, in which high-dose methotrexate-based multiagent chemotherapy 


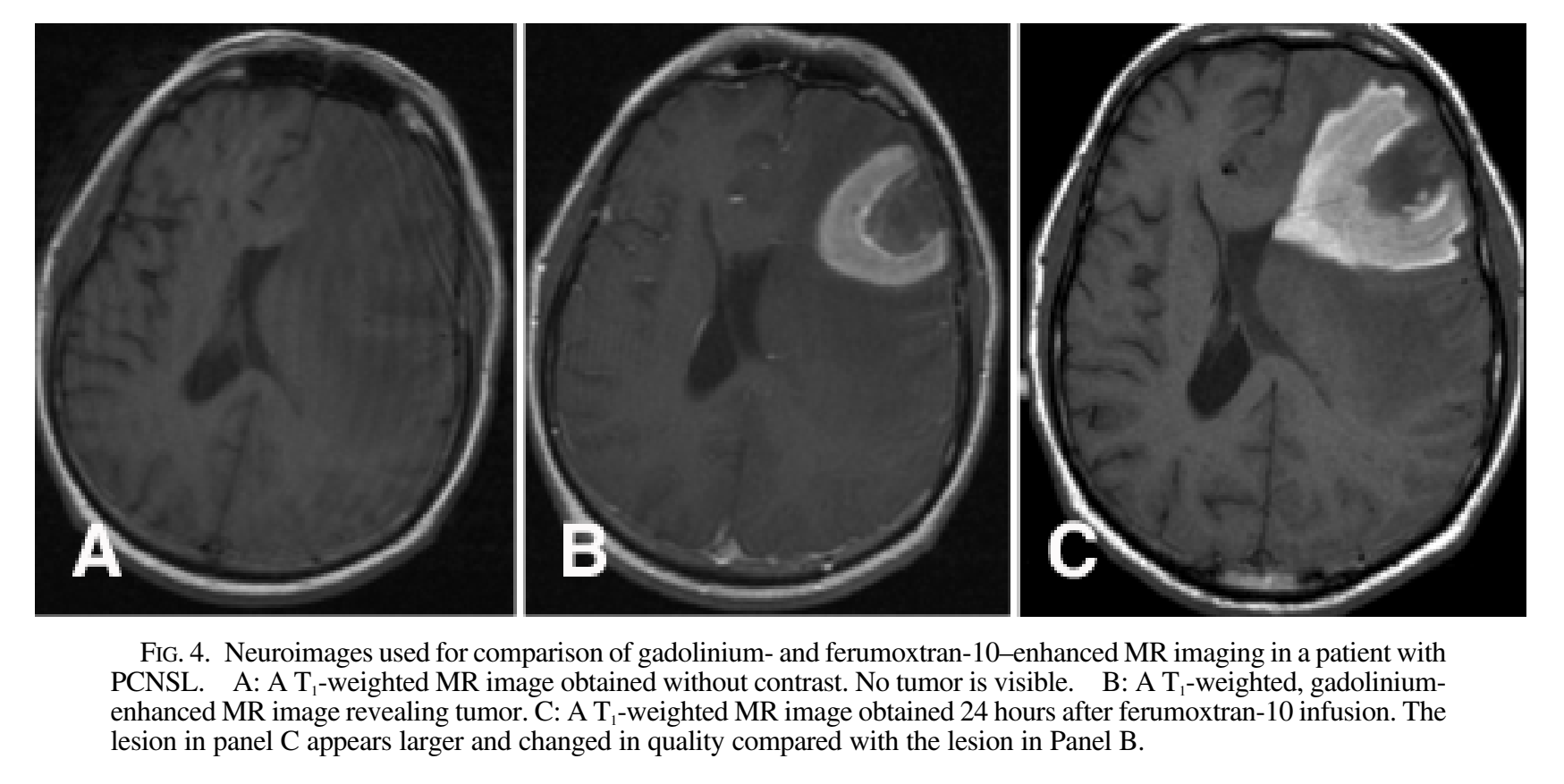

was used alone, a median survival duration of 50 months and a 5-year survival rate of $43 \%$ were achieved. However, there were six treatment-related deaths $(9 \%)$, and five of these were a result of myelosuppression (Table 1). ${ }^{66}$ Toxicity in patients treated with intraarterial methotrexatebased chemotherapy in conjunction with BBB disruption was generally manageable, and there were no procedurerelated deaths. No cases of dementia were recorded. Otherwise, the incidence of toxicities not related to the $\mathrm{BBB}$ disruption procedure is comparable to that reported in other trials on PCNSL in which chemotherapy was used alone or as part of the treatment regimen. . $^{1,5,7,16,66,67}$

The aggressive nature of PCNSL compares more to advanced-stage systemic aggressive non-Hodgkin lymphoma than to Stage $I_{E}$ lymphoma. Patients with advanced-stage systemic lymphoma are currently treated with aggressive chemotherapy, and their survival is not improved with radiotherapy. Moreover, there is no clear relationship between dose and disease outcome for radiotherapy. Furthermore, aggressive systemic non-Hodgkin lymphoma is almost invariably treated with highly active combination chemotherapy in conjunction with first-line rituximab. ${ }^{24}$ In contrast, for the aggressive neoplasm PCNSL, the mainstay of treatment is methotrexate, a drug with only moderate efficacy in lymphoma.

The use of novel approaches like monoclonal antibodies (for example, rituximab) or radioimmunotherapy (for example, ${ }^{90} \mathrm{Y}$ ibritumomab tiuxetan ${ }^{60}$ ) is hampered in PCNSL because of the impermeability of the BBB to these high-molecular-weight agents. It seems reasonable that clinical trials for new PCNSL therapies should incorporate lessons learned from the treatment of systemic aggressive non-Hodgkin lymphoma. The BBB disruption-enhanced delivery offers the opportunity to translate monoclonal antibody-based approaches and combination treatments into clinical practice for PCNSL. Monoclonal antibody-based treatment regimens and radioimmuno- therapy will be tested in the rat PCNSL model recently developed by our group. Two treatment protocols containing rituximab are currently open for enrollment at the BBB Program, one for newly diagnosed and another for relapsing PCNSL. A treatment protocol using ${ }^{90} \mathrm{Y}$ ibritumomab tiuxetan for relapsing PCNSL is currently in preparation by the BBB Group. Future plans include the establishment of a rat neurotoxicity model that should prove useful in the investigation of various factors contributing to the development of neurotoxicity as well as preventive and therapeutic strategies against this effect. The usefulness of iron oxide-based nanoparticles in the differential diagnosis of PCNSL compared with other brain lesions will be further investigated in both preclinical and clinical settings.

Further progress in the treatment of PCNSL will result from better understanding of its biological features and the performance of clinical trials. A Phase III study in which BBB disruption is compared with intravenously administered chemotherapy is desirable. However, even with committed collaboration among investigators, a randomized trial would be difficult to perform because of the rarity of PCNSL. In addition to the usual end points of survival and response rates, cognitive function and quality of life should be routine end points in all future PCNSL trials.

\section{Disclosure}

Leslie L. Muldoon (OHSU), Portland Veterans Affairs Medical Center (PVAMC), and the Department of Veterans Affairs have a significant financial interest in Adherex, a company that may have a commercial interest in the results of this research and technology. This potential conflict of interest was reviewed and managed by the OHSU Integrity Program Oversight Council and the 
PVAMC Conflict of Interest in Research Committee.

\section{References}

1. Abrey LE, Correa DD: Treatment-related neurotoxicity. Hematol Oncol Clin North Am 19:729-738, 2005

2. Abrey LE, DeAngelis LM, Yahalom J: Long-term survival in primary CNS lymphoma. J Clin Oncol 16:859-863, 1998

3. Assaf N, Hasson T, Hoch-Marchaim H, Pe'er J, Gnessin H, Deckert-Schluter M, et al: An experimental model for infiltration of malignant lymphoma to the eye and brain. Virchows Arch 431:459-467, 1997

4. Barth RF, Yang W, Rotaru JH, Moeschberger ML, Joel DD, Nawrocky MM, et al: Boron neutron capture therapy of brain tumors: enhanced survival following intracarotid injection of either sodium borocaptate or boronophenylalanine with or without blood-brain barrier disruption. Cancer Res 57: 1129-1136, 1997

5. Batchelor T, Carson K, O’Neill A, Grossman SA, Alavi J, New P, et al: Treatment of primary CNS lymphoma with methotrexate and deferred radiotherapy: a report of NABTT 96-07. J Clin Oncol 21:1044-1049, 2003

6. Blasberg RG, Patlak C, Fenstermacher JD: Intrathecal chemotherapy: brain tissue profiles after ventriculocisternal perfusion. J Pharmacol Exp Ther 195:73-83, 1975

7. Blay JY, Conroy T, Chevreau C, Thyss A, Quesnel N, Eghbali $\mathrm{H}$, et al: High-dose methotrexate for the treatment of primary cerebral lymphomas: analysis of survival and late neurologic toxicity in a retrospective series. J Clin Oncol 16:864-871, 1998

8. Bleyer WA: Leukoencephalopathy detectable by magnetic resonance imaging: much ado about nothing? Regarding Matsumoto et al. IJROBP 32:913-918; 1995. Int J Radiat Oncol Biol Phys 32:1251-1252, 1995

9. Bleyer WA: Neurologic sequelae of methotrexate and ionizing radiation: a new classification. Cancer Treat Rep 65 (1 Suppl):89-98, 1981

10. Borsi JD, Klepp O, Moe PJ: PharmCalc: Program for the calculation of clinical pharmacokinetic parameters of methotrexate. Cancer Chemother Pharmacol 22:339-343, 1988

11. Brada M, Hjiyiannakis D, Hines F, Traish D, Ashley S: Short intensive primary chemotherapy and radiotherapy in sporadic primary CNS lymphoma (PCL). Int J Radiat Oncol Biol Phys 40:1157-1162, 1998

12. Chatelut E, Roche H, Plusquellec Y, Peyrille F, De Biasi J, Pujol A, et al: Pharmacokinetic modeling of plasma and cerebrospinal fluid methotrexate after high-dose intravenous infusion in children. J Pharm Sci 80:730-734, 1991

13. Crossen JR, Goldman DL, Dahlborg SA, Neuwelt EA: Neuropsychological assessment outcomes of nonacquired immunodeficiency syndrome patients with primary central nervous system lymphoma before and after blood-brain barrier disruption chemotherapy. Neurosurgery 30:23-29, 1992

14. Dahlborg SA, Henner WD, Crossen JR, Tableman M, Petrillo A, Braziel R, et al: Non-AIDS primary CNS lymphoma: first example of a durable response in a primary brain tumor using enhanced chemotherapy delivery without cognitive loss and without radiotherapy. Cancer J Sci Am 2:166, 1996

15. DeAngelis LM, Delattre JY, Posner JB: Radiation-induced dementia in patients cured of brain metastases. Neurology 39:789-796, 1989

16. DeAngelis LM, Seiferheld W, Schold SC, Fisher B, Schultz CJ: Combination chemotherapy and radiotherapy for primary central nervous system lymphoma. Radiation Therapy Oncology Group Study 93-10. J Clin Oncol 20:4643-4648, 2002

17. DeAngelis LM, Yahalom J, Thaler HT, Kher U: Combined modality therapy for primary CNS lymphoma. J Clin Oncol 10:635-643, 1992

18. DeVita VT: Dose-response is alive and well. J Clin Oncol 4:
1157-1159, 1986

19. Doolittle ND, Miner ME, Hall WA, Siegal T, Jerome E, Osztie E, et al: Safety and efficacy of a multicenter study using intraarterial chemotherapy in conjunction with osmotic opening of the blood-brain barrier for the treatment of patients with malignant brain tumors. Cancer 88:637-647, 2000

20. Doolittle ND, Muldoon LL, Brummett RE, Tyson RM, Lacy C, Bubalo JS, et al: Delayed sodium thiosulfate as an otoprotectant against carboplatin-induced hearing loss in patients with malignant brain tumors. Clin Cancer Res 7:493-500, 2001

21. Doolittle ND, Murillo TP, Neuwelt EA: Blood-brain barrier disruption chemotherapy, in Newton HB (ed): Handbook of Brain Tumor Chemotherapy. London: Elsevier, 2006, pp 262-273

22. Doolittle ND, Petrillo A, Bell S, Cummings P, Eriksen S: Blood-brain barrier disruption for the treatment of malignant brain tumors: the National Program. J Neurosci Nurs 30: 81-90, 1998

23. Eby NL, Grufferman S, Flannelly CM, Schold SC Jr, Vogel FS, Burger PC: Increasing incidence of primary brain lymphoma in the US. Cancer 62:2461-2465, 1988

24. Feugier P, Van Hoof A, Sebban C, Solal-Celigny P, Bouabdallah R, Fermé C, et al: Long-term results of the R-CHOP study in the treatment of elderly patients with diffuse large Bcell lymphoma: a study by the Groupe d'Etude des Lymphomes de l'Adulte. J Clin Oncol 23:4117-4126, 2005

25. Frei E III, Canellos GP: Dose: a critical factor in cancer chemotherapy. Am J Med 69:585-594, 1980

26. Frei E III, Elias A, Wheeler C, Richardson P, Hryniuk W: The relationship between high-dose treatment and combination chemotherapy: the concept of summation dose intensity. Clin Cancer Res 4:2027-2037, 1998

27. Gehan EA: Dose-response relationship in clinical oncology. Cancer 54 (6 Suppl):1204-1207, 1984

28. Glantz MJ, Cole BF, Recht L, Akerley W, Mills P, Saris S, et al: High-dose intravenous methotrexate for patients with nonleukemic leptomeningeal cancer: is intrathecal chemotherapy necessary? J Clin Oncol 16:1561-1567, 1998

29. Glass J, Gruber ML, Cher L, Hochberg FH: Preirradiation methotrexate chemotherapy of primary central nervous system lymphoma: long-term outcome. J Neurosurg 81:188-195, 1994

30. Hawkey CJ, Toghill PJ: The need for prophylactic treatment to the central nervous system in patients with aggressive nonHodgkins lymphoma. Postgrad Med J 59:283-287, 1983

31. Hryniuk VM, Bertino JP: Treatment of leukemia with large doses of methotrexate and folinic acid: clinical-biochemical correlates. J Clin Invest 49:2140-2148, 1969

32. Kadan-Lottick NS, Skluzacek MC, Gurney JG: Decreasing incidence rates of primary central nervous system lymphoma. Cancer 95: 193-202, 2002

33. Keime-Guibert F, Napolitano M, Delattre JY: Neurological complications of radiotherapy and chemotherapy. J Neurol 245:695-708, 1998

34. Kimelberg HK, Kung D, Watson RE, Reiss FL, Biddlecome SM, Bourke RS: Direct administration of methotrexate into the central nervous system of primates. Part 1: distribution and degradation of methotrexate in nervous and systemic tissue after intraventricular injection. J Neurosurg 48:883-894, 1978

35. Kraemer DF, Fortin D, Doolittle ND, Neuwelt EA: Association of total dose intensity of chemotherapy in primary central nervous system lymphoma (human non-acquired immunodeficiency syndrome) and survival. Neurosurgery 48:1033-1041, 2001

36. Kraemer DF, Fortin D, Neuwelt EA: Chemotherapeutic dose intensification for treatment of malignant brain tumors: recent developments and future directions. Curr Neurol Neurosci Rep 2:216-224, 2002

37. Kroll RA, Neuwelt EA: Outwitting the blood-brain barrier for therapeutic purposes: osmotic opening and other means. 
Neurosurgery 42:1083-1099, 1998

38. Manninger SP, Muldoon LL, Nesbit G, Murillo T, Jacobs PM, Neuwelt EA: An exploratory study of ferumoxtran-10 nanoparticles as a blood-brain barrier imaging agent targeting phagocytic cells in CNS inflammatory lesions. Am J Neuroradiol 26: 2290-2300, 2005

39. McAllister LD, Doolittle ND, Guastadisegni PE, Kraemer DF, Lacy CA, Crossen JR, et al: Cognitive outcomes and long-term follow-up results after enhanced chemotherapy delivery for primary central nervous system lymphoma. Neurosurgery 46: $51-61,2000$

40. Meyers CA, Weitzner MA, Valentine AD, Levin VA: Methylphenidate therapy improves cognition, mood, and function of brain tumor patients. J Clin Oncol 16:2522-2527, 1998

41. Milano G, Thyss A, Serre Debeauvais F, Laureys G, Benoit Y, Deville A, et al: CSF drug levels for children with acute lymphoblastic leukemia treated by $5 \mathrm{~g} / \mathrm{m}^{2}$ methotrexate. A study from EORTC Children's Leukemia Cooperative Group. Eur J Cancer 26:492-495, 1990

42. Millot F, Rubie H, Mazingue F, Mechinaud F, Thyss A: Cerebrospinal fluid drug levels of leukemic children receiving $5 \mathrm{~g} /$ $\mathrm{m}^{2}$ methotrexate. Leuk Lymphoma 14:141-144, 1994

43. Nelson DF, Martz KL, Bonner H, Nelson JS, Newall J, Kerman $\mathrm{HD}$, et al: Non-Hodgkin's lymphoma of the brain: can high dose, large volume radiation therapy improve survival? Report on a prospective trial by the Radiation Therapy Oncology Group (RTOG): RTOG 8315. Int J Radiat Oncol Biol Phys 23:9-17, 1992

44. Neuwelt EA: Mechanisms of disease: the blood-brain barrier. Neurosurgery 54:131-142, 2004

45. Neuwelt EA (ed): Implications of the Blood-Brain Barrier and Its Manipulation, Vol 2. New York: Plenum Publishing Corporation, 1989

46. Neuwelt EA, Barnett PA, Bigner DD, Frenkel EP: Effects of adrenal cortical steroids and osmotic blood-brain barrier opening on methotrexate delivery to gliomas in the rodent: the factor of the blood-brain barrier. Proc Natl Acad Sci U S A 79: 4420-4423, 1982

47. Neuwelt EA, Barnett PA, Glasberg M: Pharmacology and neurotoxicity of cis-diamminedichloroplatinum, bleomycin, 5-fluorouracil, and cyclophosphamide administration following osmotic blood-brain barrier modification. Cancer Res 43: 5278-5285, 1983

48. Neuwelt EA, Barnett PA, Hellstrom I, Hellstrom KE, Beaumier P, McCormick CI, et al: Delivery of melanoma-associated immunoglobulin monoclonal antibody and Fab fragments to normal brain utilizing osmotic blood-brain barrier disruption. Cancer Res 48:4725-4729, 1988

49. Neuwelt EA, Barnett PA, Ramsey FL, Hellstrom I, Hellstrom KE, McCormick CI: Dexamethasone decreases the delivery of tumor-specific monoclonal antibody to both intracerebral and subcutaneous tumor xenografts. Neurosurgery 33:478-484, 1993

50. Neuwelt EA, Diehl JT, Vu LH, Hill SA, Michael AJ, Frenkel EP: Monitoring of methotrexate delivery in patients with malignant brain tumors after osmotic blood-brain barrier disruption. Ann Intern Med 94:449-454, 1981

51. Neuwelt EA, Frenkel E, D’Agostino AN, Carney DN, Minna JD, Barnett PA, et al: Growth of human lung tumor in the brain of the nude rat as a model to evaluate antitumor agent delivery across the blood-brain barrier. Cancer Res 45:2827-2833, 1985

52. Neuwelt EA, Frenkel EP, Gumerlock MK, Braziel R, Dana B, Hill SA: Developments in the diagnosis and treatment of primary CNS lymphoma. A prospective series. Cancer 58: 1609-1620, 1986

53. Neuwelt EA, Frenkel EP, Rapoport S, Barnett P: Effect of osmotic blood-brain barrier disruption on methotrexate pharmacokinetics in the dog. Neurosurgery 7:36-43, 1980

54. Neuwelt EA, Glasberg M, Diehl J, Frenkel EP, Barnett P: Os- motic blood-brain barrier disruption in the posterior fossa of the dog. J Neurosurg 55:742-748, 1981

55. Neuwelt EA, Glasberg M, Frenkel E, Barnett P: Neurotoxicity of chemotherapeutic agents after blood-brain barrier modification: neuropathological studies. Ann Neurol 14:316-324, 1983

56. Neuwelt EA, Goldman DL, Dahlborg SA, Crossen J, Ramsey F, Roman-Goldstein S, et al: Primary CNS lymphoma treated with osmotic blood-brain barrier disruption: prolonged survival and preservation of cognitive function. J Clin Oncol 9:1580-1590, 1991

57. Neuwelt EA, Guastadisegni PE, Várallyay P, Doolittle ND: Imaging changes and cognitive outcome in primary CNS lymphoma after enhanced chemotherapy delivery. Am J Neuroradiol 26:258-265, 2005

58. Neuwelt EA, Várallyay P, Bagó AG, Muldoon LL, Nesbit G, Nixon R: Imaging of iron oxide nanoparticles by MR and light microscopy in patients with malignant brain tumors. Neuropathol Appl Neurobiol 30:456-471, 2004

59. Neuwelt EA, Weissleder R, Nilaver G, Kroll RA, RomanGoldstein S, Szumowski J, et al: Delivery of virus-sized iron oxide particles to rodent CNS neurons. Neurosurgery 34: 777-784, 1994

60. Nowakowski GS, Witzig TE: Radioimmunotherapy for B-cell non-Hodgkin lymphoma. Clin Adv Hematol Oncol 4: 225-231, 2006

61. O'Brien P, Roos D, Pratt G, Liew K, Barton M, Poulsen M, et al: Phase II multicenter study of brief single-agent methotrexate followed by irradiation in primary CNS lymphoma. J Clin Oncol 18:519-526, 2000

62. Ohata M, Fredericks WR, Neuwelt EA, Sundaram U, Rapoport SI: [3H]Methotrexate loss from the rat brain following enhanced uptake by osmotic opening of the blood-brain barrier. Cancer Res 45:1092-1096, 1985

63. Olson JJ, Blakeley JO, Grossman SA, Weingart J, Rashid A, Supko J, et al: Differences in the distribution of methotrexate into high grade gliomas following intravenous administration, as monitored by microdialysis, are associated with blood brain barrier integrity. Proc Am Soc Clin Oncol 24 (Suppl): 1548, 2006 (Abstract)

64. O'Neill BP, Wang CH, O'Fallon JR, Colgan JD, Earle JD, Krigel RL, et al: Primary central nervous system non-Hodgkin's lymphoma (PCNSL): survival advantages with combined initial therapy? A final report of the North Central Cancer Treatment Group (NCCTG) study 86-72-52. Int J Radiat Oncol Biol Phys 43:559-563, 1999

65. Ott RJ, Brada M, Flower MA, Babich JW, Cherry SR, Deehan BJ: Measurements of blood-brain barrier permeability in patients undergoing radiotherapy and chemotherapy for primary cerebral lymphoma. Eur J Cancer 27:1356-1361, 1991

66. Pels H, Schmidt-Wolf IGH, Glasmacher A, Schulz H, Engert A, Diehl V, et al: Primary central nervous system lymphoma: results of a pilot and phase II study of systemic and intraventricular chemotherapy with deferred radiotherapy. J Clin Oncol 21:4489-4495, 2003

67. Poortmans PMP, Kluin-Nelemans HC, Haaxma-Reiche H, Van't Veer M, Hansen M, Soubeyran P, et al: High-dose methotrexate-based chemotherapy followed by consolidating radiotherapy in non-AIDS-related primary central nervous system lymphoma: European Organization for Research and Treatment of Cancer Lymphoma Group Phase II Trial 20962. J Clin Oncol 21:4483-4488, 2003

68. Rapoport SI: Blood-Brain Barrier in Physiology and Medicine. New York: Raven Press, 1976

69. Rapoport SI: Effect of concentrated solutions on blood-brain barrier. Am J Physiol 219:270-274, 1970

70. Rapoport SI, Matthews K, Thompson HK, Pettigrew KD: Osmotic opening of the blood-brain barrier in the rhesus monkey without measurable brain edema. Brain Res 136:23-29, 1977

71. Remsen LG, Marquez C, Garcia R, Thrun LA, Neuwelt EA: 


\section{Implications of the blood-brain barrier in primary CNS lymphoma}

Efficacy after sequencing of brain radiotherapy and enhanced antibody targeted chemotherapy delivery in a rodent human lung cancer brain xenograft model. Int J Radiat Oncol Biol Phys 51:1045-1049, 2001

72. Remsen LG, McCormick CI, Sexton G, Pearse HD, Garcia R, Mass M, et al: Long-term toxicity and neuropathology associated with the sequencing of cranial irradiation and enhanced chemotherapy delivery. Neurosurgery 40:1034-1042, 1997

73. Remsen LG, McCormick CI, Sexton G, Pearse HD, Garcia R, Neuwelt EA: Decreased delivery and acute toxicity of cranial irradiation and chemotherapy given with osmotic blood-brain barrier disruption in a rodent model: the issue of sequence. Clin Cancer Res 1:731-739, 1995

74. Remsen LG, Trail PA, Hellstrom I, Hellstrom KE, Neuwelt EA: Enhanced delivery improves the efficacy of a tumor-specific doxorubicin immunoconjugate in a human brain tumor xenograft model. Neurosurgery 46:704-709, 2000

75. Roman-Goldstein S, Clunie DA, Stevens J, Hogan R, Monard J, Ramsey F, et al: Osmotic blood-brain barrier disruption: CT and radionuclide imaging. Am J Neuroradiol 15:581-590, 1994

76. Roman-Goldstein S, Mitchell P, Crossen JR, Williams PC, Tindall A, Neuwelt EA: MR and cognitive testing of patients undergoing osmotic blood-brain barrier disruption with intraarterial chemotherapy. Am J Neuroradiol 16:543-553, 1995

77. Saini M, Bellinzona M, Weichhold W, Samii M: A new xenograft model of primary central nervous system lymphoma. J Neurooncol 43:153-160, 1999

78. Sandor V, Stark-Vancs V, Pearson D, Nussenblat R, Whitcup SM, Brouwers P, et al: Phase II trial of chemotherapy alone for primary CNS and intraocular lymphoma. J Clin Oncol 16: 3000-3006, 1998

79. Sarazin M, Ameri A, Monjour A, Nibio A, Poisson M, Delattre JY: Primary central nervous system lymphoma: treatment with chemotherapy and radiotherapy. Eur J Cancer 31A: 2003-2007, 1995

80. Savarese DM, Hsieh C, Stewart FM: Clinical impact of chemotherapy dose escalation in patients with hematologic malignancies and solid tumors. J Clin Oncol 15:2981-2995, 1997

81. Schabet M, Herrlinger U: Animal models of leptomeningeal metastasis. J Neurooncol 38: 199-205, 1998

82. Shapiro WR, Young DG, Mehta BM: Methotrexate: distribution in cerebrospinal fluid after intravenous, ventricular, and lumbar injections. N Engl J Med 293:161-166, 1975

83. Shenkier TN, Blay JY, O’Neill BP, Poortmans P, Thiel E, Jahnke K, et al: Primary CNS lymphoma of T-cell origin: a descriptive analysis from the International Primary CNS
Lymphoma Collaborative Group. J Clin Oncol 23:2233-2239, 2005

84. Soussain C, Muldoon LL, Varallyay C, Jahnke K, Neuwelt EA: Characterization and magnetic resonance imaging of a rat model of human B-cell central nervous system lymphoma: potential for assessing therapy and neurotoxicity. Proc Amer Assoc Cancer Res 47:2747, 2006 (Abstract)

85. Stemmler J, Schmitt M, Willems A, Bernhard H, Harbeck N, Heinemann V: Brain metastases in HER2-overexpressing metastatic breast cancer: comparative analysis of trastuzumab levels in serum and cerebrospinal fluid. Proc Am Soc Clin Oncol 24 (Suppl): 1525, 2006 (Abstract)

86. Stewart DJ, Russell N, Atack EA: Cyclophosphamide, adriamycin, vincristine, and dexamethasone in the treatment of bulky central nervous system lymphoma, in Walker MD, Thomas DGT (eds): Biology of Brain Tumours: Proceedings of the Second International Symposium on Biology of Brain Tumour (London, October 24-26, 1984). Boston: MartinusNijoff, 1986, pp 431-434

87. Thyss A, Milano G, Deville A, Manassero J, Rence N, Schneider M: Effect of dose and repeat intravenous $24 \mathrm{hr}$ infusion of methotrexate on cerebrospinal fluid availabilty in children with hematological malignancies. Eur J Cancer Clin Oncol 6: 843-847, 1987

88. Varallyay P, Nesbit G, Muldoon LL, Nixon RR, Delashaw J, Cohen JI, et al: Comparison of two superparamagnetic viralsized iron oxide particles ferumoxides and ferumoxtran-10 with a gadolinium chelate in imaging intracranial tumors. Am J Neuroradiol 23:510-519, 2002

89. Walker MD, Weiss HD: Chemotherapy in the treatment of malignant brain tumors. Adv Neurol 13:149-191, 1975

90. Zubair AC, Ali SA, Rees RC, Goepel JR, Winfield DA, Goyns $\mathrm{MH}$ : Analysis of the colonization of unirradiated and irradiated SCID mice by human lymphoma and non-malignant lymphoid cells. Leuk Lymphoma 22:463-471, 1996

Studies reported in this review were supported by National Institutes of Health Grant Nos. NS34608, NS33618, and NS44687. Manuscript received August 11, 2006.

Accepted in final form October 4, 2006.

Address reprint requests to: Edward A. Neuwelt, M.D., Department of Neurology, Blood-Brain Barrier and Neuro-Oncology Program, Oregon Health \& Science University, 3181 Sam Jackson 